\title{
Numerical modelling and comparison analysis of pressure distribution in the gas film for non-contacting face seals
}

\author{
Slawomir Blasiak ${ }^{1, *}$ \\ ${ }^{1}$ Kielce University of Technology, Faculty of Mechatronics and Mechanical Engineering, \\ Department of Mechanical Technology and Metrology, Aleja Tysiaclecia Panstwa Polskiego 7, 25-314 Kielce, Poland
}

\begin{abstract}
Summary. The tightened-up norms concerning the emission of harmful substances into the natural environment are putting before designers of knots sealing very rigorous requirements. One should notice that mechanical seals are ones of most often applied sub-assemblies of the sealing nodes. The main task for mechanical seals is to maintain maximum tightness. They are applied practically in all branches of industry and must fulfil their function while working under various operating conditions. The determination of the pressure distribution in the fluid film layer is necessary to calculate the gasdynamic force and moments. A two-dimensional equation of Reynolds was solved based on Finite Volume Method (FVM). At the presented work findings of the simulation results concerning the comparison get based on the author's computer program in $\mathrm{C}++$ language with results obtained from the commercial ANSYS CFX program was presented.
\end{abstract}

\section{Introduction}

When reviewing the references related to sealing and lubrication technology, one may distinguish a series of papers concerning mechanical seals, especially noncontacting face seals. The basic reason for this is a series of significant problems that must be solved in order to sufficiently investigate physical phenomena occurring within flow-through machines and directly in mechanical seals. Another reason is aggravating standards concerning emission of harmful substance to the natural environment. This forces designers to improve the existing or search for new structural solutions. As is generally known, mechanical seals are trouble spots of machines and devices in which they operate. Seals such as these are commonly used in all sectors of industry and operate under various operation and environmental conditions. The most important task put before them is separation of sealed media from the environment. In almost all existing designs of mechanical seals, the same problem applies to maintaining clearance separating the working rings at set level ca. $1 \mu \mathrm{m}$. In case of noncontacting gas face seals, one of the methods that allows for keeping stable layer of medium, is introduction of different geometrical modifications (microstructures) on the working rings races. This can be radial or spiral grooves $[1,2]$ or textured face surface $[3,4]$ and the others parameters $[5,6]$. Change of the faces of sealing rings entails changes of the fluid film dynamic properties and temperature [7-9]. Such properties are described using rigidity and dampening factors which in turn indirectly determine the force generated in the layer separating the rings. Keeping the balance of forces affecting the rings array allows for maintaining stable film of medium and precludes rings contact during operation. Results of the studies concerning impact of the geometry changes of the applied faces modification on the fluid film dynamic properties are presented in the papers, e.g. [10-14]. Such analyses enable to determine, among other things, which of the discussed parameters have a key impact on the properties in question. In the papers of [15-19], the authors present results of numerical analyses of the mathematical model covering ring vibration dynamics equations and non-linear Reynolds equation for compressible medium. Solutions of the Reynolds equation and determination of pressure distribution in the fluid film is crucial during calculation of hydrodynamic forces and moments. Due to this fact, determination of pressure distribution within radial clearance represents an important scientific problem.

\section{Subject of research}

Based on the papers of [20,21], Fig. 1 presents an example of non-contacting gas face seal.

Non-contacting gas face seal, consists of two rings. Stator (1) is flexibly set in the case and has open radial grooves (3) made on the periphery of its face surface.

* Corresponding author: sblasiak@tu.kielce.pl 


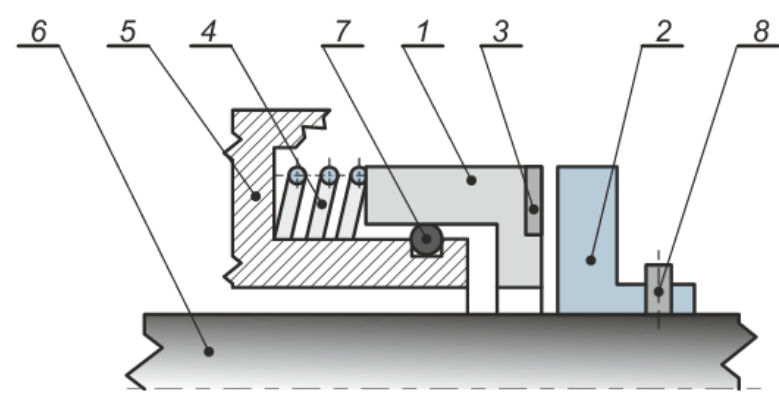

Fig. 1. Schematic cross section drawing of a non-contacting gas face seal; 1 - stator, 2 - rotor, 3 - groove, 4 - spring, 5 casing, 6 - shaft, 7 - O-ring, 8 - steady pin.

They are oriented towards the space on the process side (i.e. throttle) filled with sealed off medium of a given pressure Po. However, rotor (2) rotates together with the shaft (6) of the rotor machine.

\section{Mathematical model}

Normal height of the radial clearance $h_{o}$, results from the balance of forces affecting the arrangement of working rings and it is designed at the designing stage. It depends on many factors: operating conditions (rotational speed and differential pressure), thermal and physical properties of the sealed medium, properties on materials used for rings, geometry itself of the sealing rings, thermoelastic deformations, temperature, etc. In the case in question, the following assumption was made $h_{o}=1 \mu m$.

Applying the generally known simplifying assumptions, motion equations and continuity equation were reduced to a single equation describing pressure distribution within the radial clearance - so called Reynolds equation [16,21].

$$
\vec{\nabla}\left[p h^{3} \vec{\nabla} p-6 \mu \omega r p h \vec{e}_{\theta}\right]=12 \mu \frac{\partial(p h)}{\partial t}
$$

Determination of pressure distribution was possible having assumed the boundary conditions and periodicity condition in the following form:

$$
\begin{gathered}
\left.p(r, \theta)\right|_{r=r_{i}}=p_{i} ;\left.p(r, \theta)\right|_{r=r_{o}}=p_{o} \\
\left.p(r, \theta)\right|_{\theta=0}=\left.p(r, \theta)\right|_{\theta=2 \pi}
\end{gathered}
$$

\section{Reynolds equation - numerical solution}

In the presented paper, two-dimensional Reynolds equation (1) was solved using numerical methods. The most often used methods are as follows: Finite Volume Method (FVM), Finite Element Method (FEM) [20] and the Finite Difference Method as the less often used method. Correct determination of pressure distribution, especially within a clearance of complicated topography determines achievement of more accurate values of (hydro) gasdynamic forces and moments. Diagram of the calculation grid for the finite volume method is presented on the below figure.

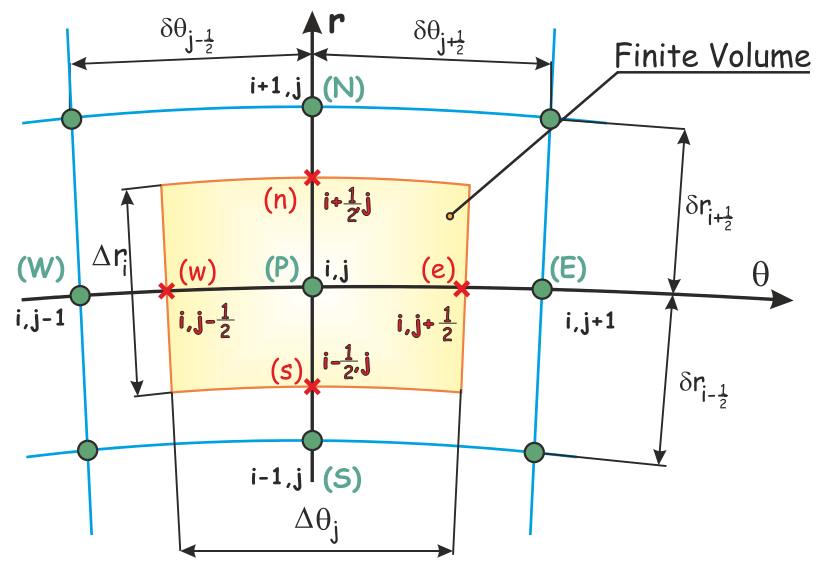

Fig. 2. Diagram of the finite volume grid.

In the presented calculation algorithm using the method of elementary control volume, the equation (1) was solved performing the integration on the elementary control volume of each of the equation components, as expressed by:

$$
\begin{aligned}
12 \int_{s}^{n} \int_{w}^{e} \mu r h \frac{\partial p}{\partial t} d r d \theta=\int_{s}^{n} \int_{w}^{e} \frac{\partial}{\partial r}\left(p h^{3} r \frac{\partial p}{\partial r}\right) d r d \theta \\
+\int_{s}^{n} \int_{w}^{e} \frac{\partial}{\partial \theta}\left(\frac{p h^{3}}{r} \frac{\partial p}{\partial \theta}-6 \mu \omega r p h\right) d r d \theta \\
-12 \int_{s}^{n} \int_{w}^{e} \mu r p \frac{\partial h}{\partial t} d r d \theta
\end{aligned}
$$

As a result, one obtained:

$$
p_{P}=\frac{A_{N} p_{N}+A_{S} p_{S}+A_{E} p_{E}+A_{W} p_{W}}{\left(A_{N}+A_{S}+A_{E}+A_{W}+F_{e}-F_{w}+\bar{S}\right)}
$$

where:

$$
\begin{gathered}
A_{N}=D_{n} ; A_{S}=D_{s} \\
A_{E}=D_{e}+\max \left(0,-F_{e}\right) \quad A_{W}=D_{w}+\max \left(F_{w}, 0\right) \\
D_{n}=\left(\frac{p h^{3} r \Delta \theta}{\Delta r}\right)_{n} ; D_{s}=\left(\frac{p h^{3} r \Delta \theta}{\Delta r}\right)_{s} ; \\
D_{e}=\left(\frac{p h^{3} \Delta r}{r \Delta \theta}\right)_{e} ; D_{w}=\left(\frac{p h^{3} \Delta r}{r \Delta \theta}\right)_{w} \\
F_{e}=(6 \mu \omega r h \Delta r)_{e}, F_{w}=(6 \mu \omega r h \Delta r)_{w}, \\
\bar{S}=\left(12 \mu r \frac{\partial h}{\partial t} \Delta r \Delta \theta\right)
\end{gathered}
$$

The main drawback of the presented central differential diagram (equation (4)) is its dependence on flow direction. In order to improve stability of the solution, the upwind differencing scheme was introduced, general form of which is presented in the form of the dependency (6). In the developed proprietary computer software, a grid of span 80 × 80 mesh was adopted for numerical calculations. This density of calculating grid mesh results from the simplicity of 
geometry of the applied modifications. The used geometry of radial grooves is deprived of curved elements like in case of spiral grooves, and all edges of the applied modifications run along the conventional lines connecting individual grid nodes. In the author's opinion, application of the calculation grid of too small mesh size, despite significant calculation power of contemporary computers, may lead to groundless extension of numerical calculation time.

\section{FEM method}

In order to perform the comparative studies using commercially available software ANSYS CFX, one modelled the radial clearance identical as in the case of the software developed by the author.

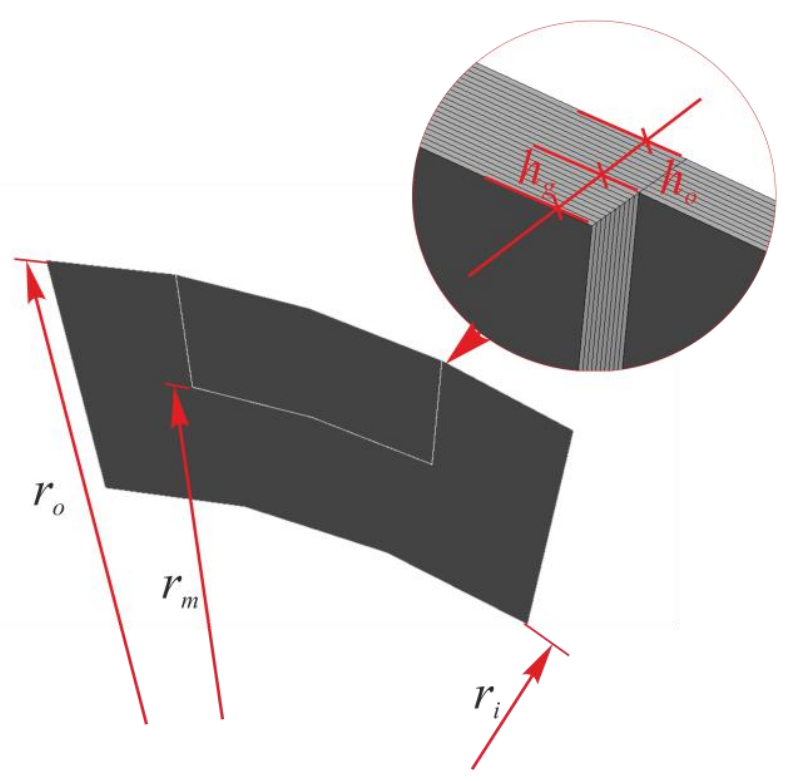

Fig. 3. Radial clearance geometry.

Calculation grid was implemented, as shown on the figure below.

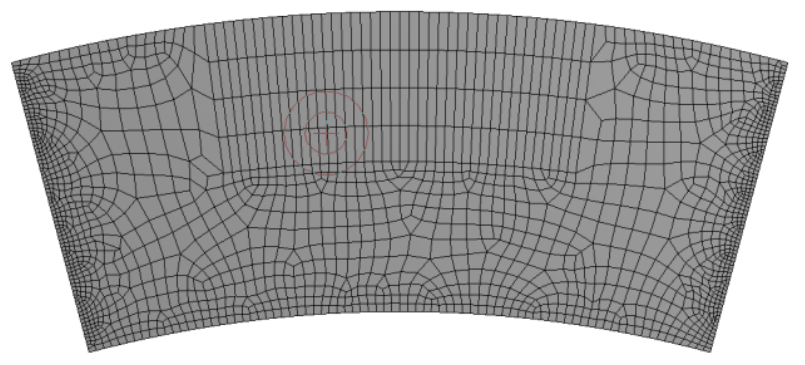

Fig. 4. Finite element mesh.

Spatial division of the calculation grid was performed as follows: the boundaries towards radial and circumferential direction on 80 parts and towards axial direction on 10 parts of the clearance height and ten parts of the radial groove depth.

\section{Results and discussion}

The mathematical model presented in section 3 was numerically solved using author's computer software in $\mathrm{C}++$ language.

Table 1. Gas face seal performance parameters.

\begin{tabular}{|c|c|}
\hline Parameter & Value \\
\hline Shaft angular velocity & $\omega=2000[\mathrm{rad} / \mathrm{s}]$ \\
\hline Gas viscosity & $\mu=1.8\left(10^{-5}\right)[\mathrm{Pas}]$ \\
\hline Design clearance & $h_{o}=6\left(10^{-6}\right)[\mathrm{m}]$ \\
\hline Groove depth & $h_{g}=6\left(10^{-6}\right)[\mathrm{m}]$ \\
\hline Pressure on the inner radius & $p_{i}=1\left(10^{5}\right)[\mathrm{Pa}]$ \\
\hline Pressure on the outer radius & $p_{o}=2\left(10^{5}\right)[\mathrm{Pa}]$ \\
\hline Inner radius & $r_{i}=0.048[\mathrm{~m}]$ \\
\hline Outer radius & $r_{o}=0.060[\mathrm{~m}]$ \\
\hline Groove radius & $r_{g}=r_{m}=0.5\left(r_{i}+r_{o}\right)$ \\
\hline Number of grooves & $n_{g}=6$ \\
\hline
\end{tabular}

Obtained results of the pressure distribution are presented below and shown on a (fragment) section of the sealing ring towards circumferential direction, representing a single segment.

a)

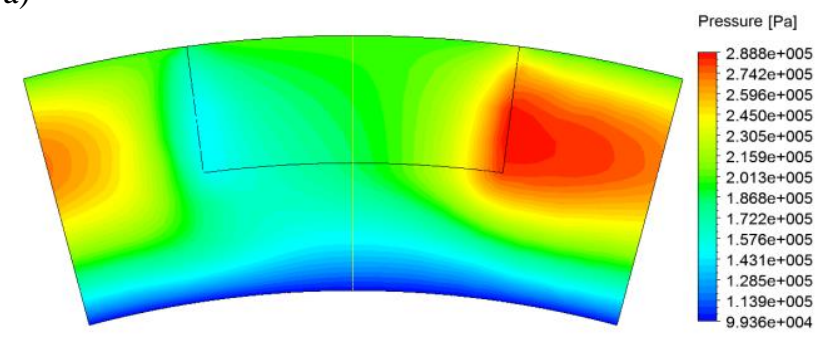

b)

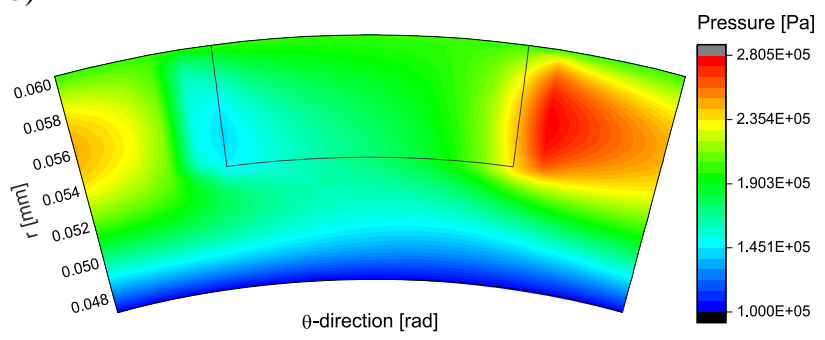

Fig. 5. Pressure distribution a) Ansys CFX, b) author's C++ language computer software.

Obtained results were compared with the results obtained using ANSYS CFX software. All calculations were performed based on parameters given in table 1 .

Reynolds number in the gas face seal is calculated from eq. (7) as defined by $[22,23]$ :

$$
\operatorname{Re}=\frac{\rho \omega r_{m} h}{\mu}
$$


The Reynolds number is 77.96 for Ansys computation and 77.34 for author's computer programme.

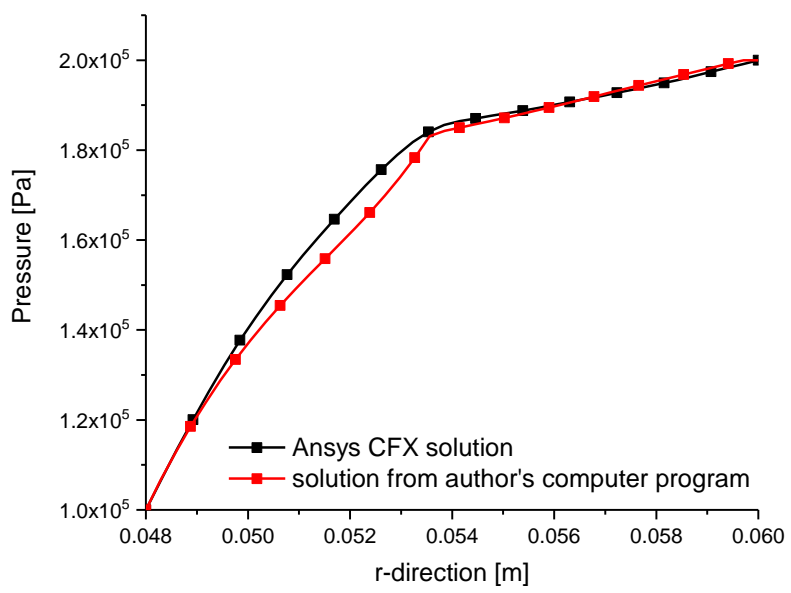

Fig. 6. Pressure distribution - results comparison.

When analysing the results from figure Fig. 5 and Fig. 6 one may observe significant conformity of the obtained results. Minor differences may result from a fact that in the commercial software, flow of factor is described using Navier-Stokes equations without any simplifying assumptions used in case of the presented model of flow for non-contacting face seals. Another reason may be the fact that as a result of solving the Reynolds equation, one gets averaged value of pressure along the radial clearance height.

\section{Conclusions}

When performing the analysis of the obtained results, one finds significant consistency of the determined pressure distributions in the radial clearance. This is very important especially in the case of the race of working rings with modified surfaces, wherein while solving simultaneous equations describing rings vibrations, it is necessary to determine forces and moments that depend directly on pressure. Application of differentiated surface topography positively affects seals operation. This results in clear reduction of rings operating (sliding) surfaces wearing with simultaneous minimization of leakage and friction resistance. Achievement of these results would not be possible without performing oriented scientific studies both laboratory and practical on workstations specially designed for that purpose. This is however related to significant financial expenditures and is labour consuming as well as necessitates performance of numerous experimental studies within this range. The alternative for experimental studies is development of a calculation apparatus supporting the designing stage, based on more and more accurate mathematical models describing multidisciplinary issues within the scope of fluid mechanics, continuum mechanics or mechanical systems dynamics. Therefore, it is necessary to develop specials programs that would allow for performing complex analyses and simulation studies concerning non-contacting face seals with modified surfaces.

\section{References}

1. R. A. Shellef, R. P. Johnson, Tribol. Trans. 35, 53 (1992)

2. N. Zirkelback, Tribol. Trans. 43, 337 (2000)

3. Y. Feldman, Y. Kligerman, I. Etsion, Tribol. Lett. 22, 21 (2006)

4. B. Antoszewski, E. Evin, J. Audy, J. Tribol. 130, 21303 (2008)

5. L. Nowakowski, M. Skrzyniarz, E. Miko, J. Takosoglu, S. Blasiak, P. Laski, G. Bracha, D. Pietrala, J. Zwierzchowski, M. Blasiak, eds., Influence of the cutting parameters on the workpiece temperature during face milling (The European Physical Journal Conference, 2016)

6. J. Takosoglu, P. Laski, S. Blasiak, G. Bracha, D. Pietrala, J. Zwierzchowski, L. Nowakowski, eds., Determination of flow-rate characteristics and parameters of piezo pilot valves (The European Physical Journal Conference, 2016)

7. B. Maciejewska, M. Piasecka, HEAT AND MASS TRANSFER 53, 1211 (2017)

8. B. Maciejewska, M. Piasecka, Int. J. Heat Mass Transfer 107, 925 (2017)

9. K. Strak, M. Piasecka, B. Maciejewska, Int. J. Heat Mass Transfer 117, 375 (2018)

10. B. A. Miller, I. Green, J. Tribol. 123, 395 (2001)

11. B. A. Miller, I. Green, J. Tribol. 124, 755 (2002)

12. I. Green, R. M. Barnsby, J. Tribol. 124, 151 (2002)

13. B. A. Miller, I. Green, J. Tribol. 125, 403 (2003)

14. B. Wang, CJME 24, 146 (2011)

15. D. Bonneau, J. Huitric, B. Tournerie, J. Tribol. 115, 348 (1993)

16. I. Green, R. M. Barnsby, J. Tribol. 123, 388 (2001)

17. B. Tournerie, N. Brunetiere, J.-C. Danos, Sealing Technol. 2003, 10 (2003)

18. S. Blasiak, Int. J. Heat Mass Transfer 100, 79 (2016)

19. S. Blasiak, ed., Numerical analysis of the noncontacting gas face seals (IOP Conference SeriesMaterials Science and Engineering, 2017)

20. B. Ruan, Tribol. Trans. 43, 411 (2000)

21. B. Ruan, J. Tribol. 124, 186 (2002)

22. B. Wang, H. Zhang, H. Cao, CJME 26, 78 (2013)

23. I. Shahin, J Aeronaut Aerospace Eng 05 (2016) 